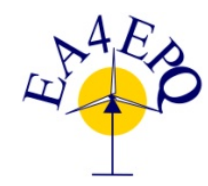

International Conference on Renewable Energies and Power Quality (ICREPQ'17)

Malaga (Spain), $4^{\text {th }}$ to $6^{\text {th }}$ April, 2017

Renewable Eenergy and OPurer Quality. Yournal (RE\&PQJ)

ISSN 2172-038 X, No.15 April 2017

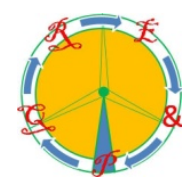

\title{
Least-squares versus LMS parametric approaches for power quality events segmentation
}

\author{
Enrique Alameda-Hernandez, Fernando Aznar, Francisco Gil*, Antonio Espin \\ Área de Ingeniería Eléctrica \\ Campus Fuentenueva. Universidad de Granada. CP 18071. Granada. Spain. \\ e-mails: \{ealameda,faznar, aespin\}@ugr.es \\ *Área de Ingeniería Eléctrica \\ Universidad de Almería. CP 04120. Almería. Spain. \\ e-mail: pagilm@ual.es
}

\begin{abstract}
Power quality monitoring requires knowing when the start of the perturbation takes place, and also when it ends; in this way, the voltage or current signals are divided into segments. In this work, we follow previously developed ideas in the literature and resort to parametric modelling to achieve the perturbed signal segmentation. What we propose here is the use of adaptive AR modelling identification, in particular Recursive Least Squares and Least Mean Squares, as opposed to a block-based approach used elsewhere. Overdetermined systems, both block-wise and adaptively are also included among the analysed methods. Simulations show that although being computationally lighter, and hence more suitable to real-time implementations, segments limits are accurately located by adaptive algorithms most of the cases.
\end{abstract}

\section{Key words}

Power quality, perturbations, segmentation, adaptive algorithm, parametric modelling.

\section{Introduction}

A healthy power system can be identified, among other facts, by the quality of the power that it is delivering. Any deviation of the rated voltage, either in rms value or frequency, for instance, is termed as a perturbation. Power perturbations affect both the power grid itself and the loads connected to it, so a proper monitoring of the delivered power is crucial, specially the detection of a perturbation at the same instant that it appears. This operation must be carried out in real time, allowing allocating more computational resources to identifying and characterising in proper detail this perturbation, and eventually applying the healing measures that may be required. In order to achieve this segmentation of the voltage or current signals into healthy and unhealthy segments, signal processing methods are applied $[1,2]$. In particular, an autorregresive (AR) parametric model is fitted to the signal of interest during a given block of samples, so the modelling error is small. If the same model is applied to the next block of samples, two things can come out. First, the signal behaviour has not changed, so the model is still valid and the modelling error is small. Second, the signal behaviour has deviated from the previous block, so the model error increases. This increment can be used to detect this change in behaviour, either the perturbation start, end or change.

The block-wise approach is required here, since some kind of adaptivity is necessary -i.e. as new samples arrive, the algorithm is able to provide new information. Nevertheless, it may not be the best adaptive approach, since it is computationally demanding, since basically each block is solved without considering any previous knowledge that could have been computed in any previous block. In this sense, it is very interesting to consider other kinds of adaptivity implementations. One of such implementations is found when the signal is recursively fitted to the AR model through least-squares, using the so called Recursive Least Squares (RLS) algorithm. Although not for segmentation, RLS algorithms have been previously used in the power quality context. In [3], a novel RLS type algorithm is compared against other RLS and LMS 
algorithms when applied to harmonic parameters estimation. Likewise, in [4] a similar analysis is carried out but for a limited number of algorithms. Another feasible adaptive implementation to solve the segmentation problem is based on finding the least mean square (LMS) of the modelling error, via the so called LMS algorithm. Compared to RLS algorithms, LMS algorithms are computationally lighter, so in this sense, are better candidates for real-time implementation, and hence are worth considering. LMS algorithms were analysed by the authors previously [5], but not compared to any other pure adaptive algorithm, like the RLS here. Finally, due to the estimation process inherent in this kind of problems, where in some cases statistical magnitudes are computed from time averages of signal samples assuming ergodicity [6], considering more equations than model order parameters - that are the unknowns in this problem - is likely to yield more accurate results. Based on this idea, overdetermined implementations of the methods discussed in the previous paragraphs are also considered.

\section{Solving parametric models}

Parametric models are those which definition depends only on the value of a certain number of parameters. One of the most general one is the autoregressive moving-average (ARMA), but here we concentrate only on its AR part, so a signal $y(n)$ follows an AR model if

$$
y(n)=-\sum_{i=1}^{p} a_{i} y(n-i)+w(n)
$$

where $\left\{a_{i}\right\}_{i=1}^{p}$ are the model parameters and $\{w(n)\}$ is an independent and identically distributed zero mean process (noise). If both sides of (1) are multiplied by $y(n-k)$ and then the expected value operator $\mathrm{E}[\cdot]$ is applied, the Yule-Walker equations are obtained:

$$
\mathrm{E}[y(n) y(n-k)]=-\sum_{i=1}^{p} a_{i} \mathrm{E}[y(n-i) y(n-k)] .
$$

These expected values for different $k$ and $i$ constitute the autocorrelation sequence of process $y(n)$. Assuming ergodicity, they can be estimated by sample averaging, so (2), including estimated expected values, provides estimated values of the model parameters, $\left\{\hat{a}_{i}\right\}$. For the application in mind, one would expect that a good model fit would result in a small $w(n)$ in (1).

\section{A. Adaptive algorithms}

An adaptive approach to a problem resolution serves two main purposes. First one, at the arrival of new information - i.e. new data - update the result. Second one, track the time evolution of the system, continuously updating the gathered information and hence the result. The second one is of interest here. A well known and simple adaptive algorithm is the LMS. This algorithm can impose an orthogonality condition between a vector of data $\mathbf{x}(n)$ and the estimation error $e(n)$, that is $\mathrm{E}[\mathbf{x}(n) e(n)]=0$ [7]. Remarkably, defining $\mathbf{x}(n)=-[y(n-1), y(n-1), \ldots y(n-N)]^{T}, d(n)=y(n)$ and $e(n)=d(n)-\mathbf{a}^{T} \mathbf{x}(n)$, the Yule-Walker equation (2) shows up. LMS can then be used to estimate the AR parameters vector $\mathbf{a}=\left[a_{1}, a_{2}, \ldots, a_{p}\right]^{\mathrm{T}}$. Further, $e(n)=w(n)$.

Since the orthogonality condition arises from the minimization of error signal $e(n)$, any other algorithm that minimises it would solve the YW equations provided the variables are defined as in the previous paragraph. One option is to follow a least-squares approach, like the Recursive Least Squares (RLS) algorithm, [7].

\section{B. Overdetermined algorithms}

Provided that the problem at hand is an estimation one, it could seem reasonable to include as many equations as possible in order to improve the process. YW equations are a set of linear equations described in (2), for $k=1, \ldots, p$. But an overdetermined system can be obtained if $k$ takes longer values, say up to $2 p$. Such an overdetermined system can be solved using the pseudoinverse of the linear system matrix. Adaptive implementations are also possible for overdetermined systems. An extension of the RLS algorithm to this case is the Overdetermined Recursive Instrumental Variable (ORIV) algorithm. Likewise, for LMS the Averaged Overdetermined and Generalised LMS (AOGLMS) is available [8].

\section{Examples and simulations}

In order to illustrate and later compare the described approaches to compute AR models, four different power quality perturbations will be considered:

P1.- An $0.8 \mathrm{pu}$ voltage sag.

P2.- An 0.8 pu voltage sag, with two step recovery.

P3.- A 1.2 pu voltage swell.

P4.- Voltage transient, following section 8.10.1.1 of [1].

Perturbations are computer generated, assuming a sampling frequency of $6.4 \mathrm{kHz}$ and a duration of 1792 samples. Small Gaussian noise is added, at $25 \mathrm{~dB}$ SNR. Performances of these methods under $\mathrm{P} 1-\mathrm{P} 4$ perturbations follow. 


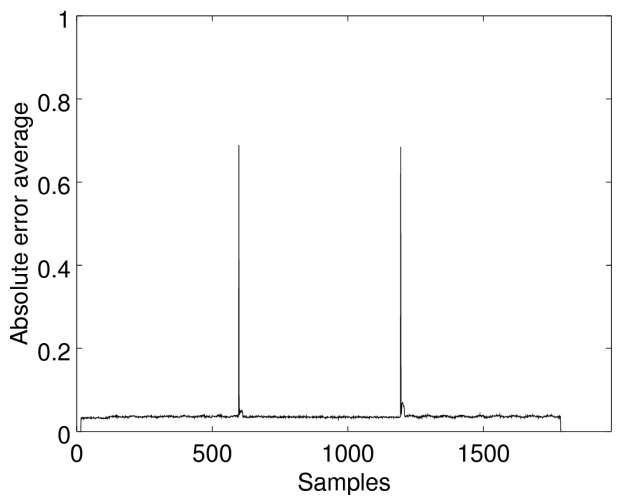

Fig. 1- Error signal from YW equations, solved block-wise. Best parameter combination shown: $p=15, B l=200$ and $o v l=50 \%$.

\section{A. Sudden change caused by a voltage sag}

The synthetic sag starts at sample 598, and finishes at 1195. At these limiting points, voltages changes from $-0.86 \mathrm{pu}$ to $-0.18 \mathrm{pu}$ and from $0.18 \mathrm{pu}$ to $0.86 \mathrm{pu}$, respectively. So, the perturbation is easily locally located. By locally, we mean that from a short observation window, it can be said that something is changing in the signal, and hence the described methods will have no real problem.

Let us first consider the error signals provided by the YW equations solved block-wise, obtained for a range of model orders, block lengths $B l$ and overlapping $o v l$. Peaks are nicely well defined (a line in fact) with no significant difference in heights, while base error oscillates for lower block lengths. Best results - i.e. higher peaks - are obtained for higher model orders, longer lengths and overlapping, see figure 1 . When an overdetermined system is formed using the YW equations, irregular behaviour of $e(n)$ producing some huge peaks for some parameters combination happens. Can this irregularity be caused by the computation of the pseudoinverse? Peak location is quite accurate, within a range of 3-4 samples at the most. Best performance is shown in figure 2 .

Applying the LMS formalism to the YW equations results in smooth and regular $e(n)$. Some analysed parameter combinations make the algorithm diverge, so a study to determine the valid parameters range is required, in particular that of the step-size $\mu$. Disregarding the initial converge samples, the rest of combinations - the non-diverging ones - provide good results: perfect first and second peak location, being $\mu=0.05$ the best option, with little difference for $p=5,10,15$ (see figure 3). Regarding an overdetermined LMS approach to YW equations, it brings more diverging parameter combinations, being $e(n)$ oscillatory during

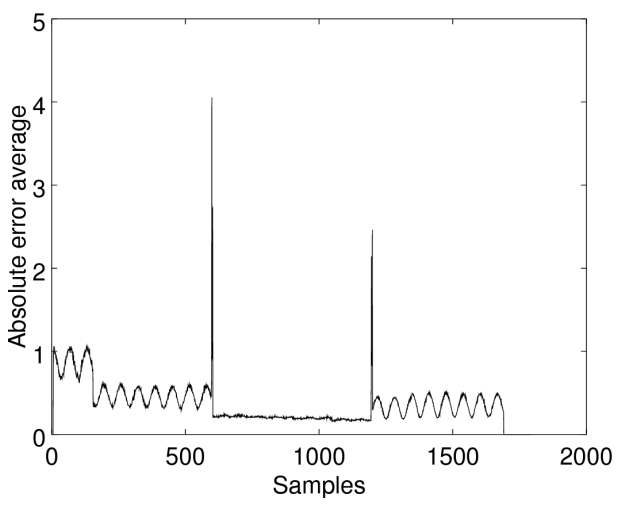

Fig. 2- Error signal from overdetermined YW equations, solved block-wise. Best parameter combination shown: $p=$ $5, B l=200$ and $o v l=25 \%$. Note the difference in scale compared to figure 1 .

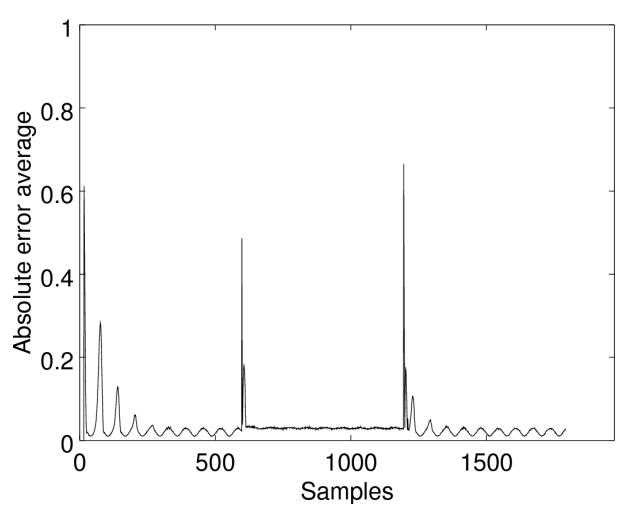

Fig. 3- Error signal from YW equations, adaptively solved (LMS). Best parameter combination shown: $p=15, \mu=$ 0.05 .

convergence and base level. As figure 4 shows, compared to figure 3 , convergence is slower, but provides higher peaks than non-overdetermined LMS. When the ORIV algorithm is employed, all analysed parameters combinations exactly locate the start and end of the sag. Nevertheless, higher peaks are provided by $p=5$ and forgetting factor $\lambda=0.99$, while best defined peaks by $p=10$ and $\lambda=0.99$, please refer to figure 5 . Finally, for the RLS algorithm an excellent definition is achieved, with low smooth base level and sharp, correctly located, peaks, for all parameters combinations (see figure 6). In order to allow a better comparison of the presented algorithmic results so far, in table I, the location of the peak and its height is presented for each case. Recall that for adaptive algorithms, the initial samples of signal $e(n)$ are not considered since the algorithms have not fully converged yet. As clearly seen, the overdetermined block-wise approach is the algorithm that show a more distinctive behaviour since, unlike the rest of algorithms the estimated peaks are slighted (4 samples) deviated from the exact location, but the heights are well above the rest. Aside this par- 


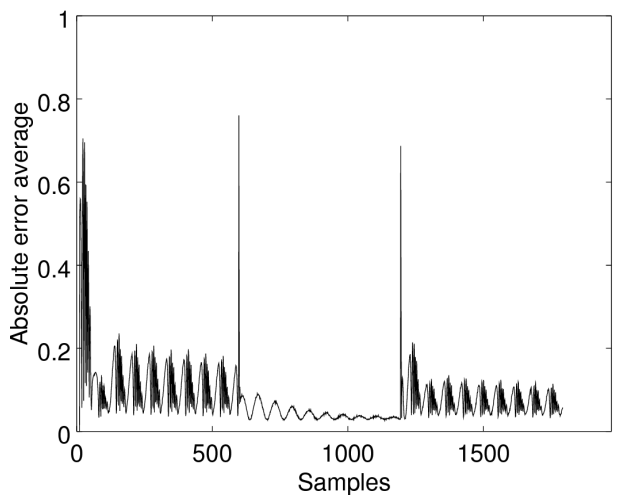

Fig. 4- Error signal from overdetermined YW equations, adaptively solved (LMS). Best parameter combination shown: $p=15, \mu=0.15, f=0.94$.

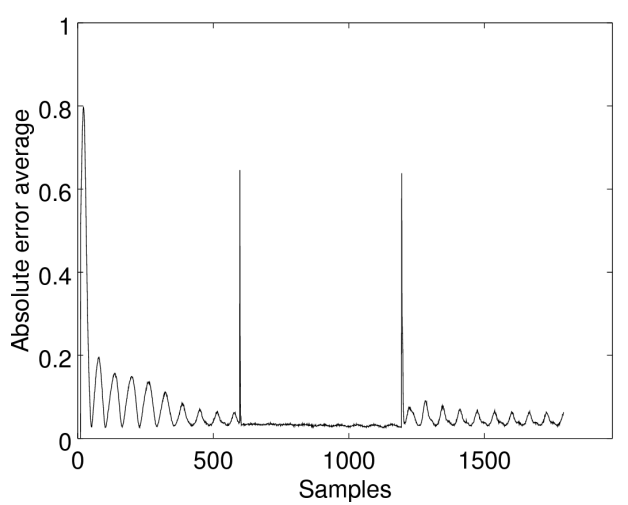

Fig. 5- Error signal from overdetermined YW equations, adaptively solved (ORIV). Best parameter combination shown: $p=15, \lambda=0.99$.

ticular case, the overdetermined LMS algorithm provides the best results of all studied cases, since the peak location is exact and the peaks are the highest.

\section{B. Soft change in a (two step) voltage sag}

The second perturbation that was considered was a two step sag, where the first sag is $0.8 \mathrm{pu}$, as in the previous example, but then the sag is fully recover just after going through a lesser $(0.4 \mathrm{pu})$ gap. Under this situation, one may expect an identical behaviour to the previous example in what respect the identification of the beginning of the sag, but surprisingly, it is not so. Only a few methods do the work:

a) Block-wise approach: only larger $p$ with longer block lengths are able to correctly locate the three transition points, and it should be noted that the peaks are 10 times lower than for the single step sag case, and hence the difficulty in a correct detection.

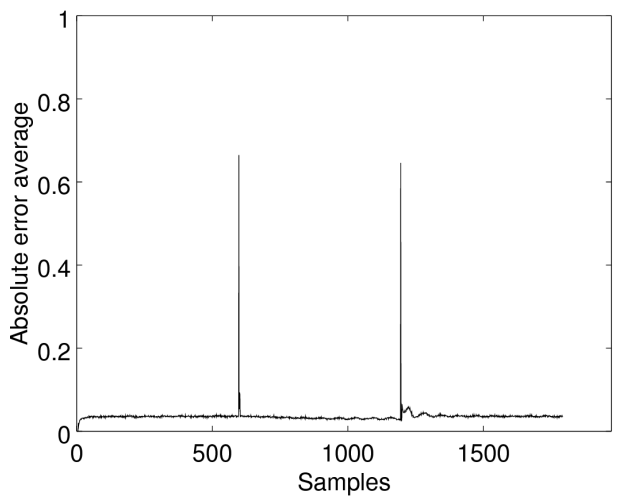

Fig. 6- Error signal from YW equations, adaptively solved (RLS). Best parameter combination: $p=15, \lambda=0.99$.

Table I- Peaks location (in samples) and heights obtained during the segmentation of the sag, for all the analysed approaches. Exact peak location, 598 and 1195 samples respectively for peak 1 and 2 .

\begin{tabular}{|c|c|c|c|c|}
\hline Method & peak 1 & height 1 & peak 2 & height 2 \\
\hline Block-wise & 598 & 0.689 & 1195 & 0.685 \\
\hline $\begin{array}{c}\text { Overdetermin } \\
\text { ed block-wise }\end{array}$ & 599 & 4.04 & 1199 & 2.47 \\
\hline LMS & 598 & 0.485 & 1195 & 0.664 \\
\hline $\begin{array}{c}\text { Overdeterm } \\
\text { ined LMS }\end{array}$ & 598 & 0.762 & 1195 & 0.689 \\
\hline ORIV & 598 & 0.645 & 1195 & 0.636 \\
\hline RLS & 598 & 0.664 & 1195 & 0.646 \\
\hline
\end{tabular}

b) LMS approach: the error signal $e(n)$ has a regular behaviour, but again the peaks are quite small.

c) RLS approach: as for the one step sag, excellent peak definition, although being smaller. Nevertheless, it carries a small delay of 4 samples.

The reason behind the small peaks is that although the first step decreases in $0.8 \mathrm{pu}$ (like in the simple one step sag) the signal is smoother since the step happens to be in a zero crossing. So, smooth transitions require longer model order and block lengths, so that the scope is widen and changes are spotted. For a fair comparison, all three working methods are shown in figure 7. From it, it is clear that best behaviour is shown by the RLS algorithm, since it is smooth with sharp peaks, followed by the LMS algorithm; finally, the standard block-wise approach provides the poorest result. Overall, note that the peaks are now much shorter, compared with the one step sag analysed in section 3.A.

\section{Other perturbations}

Besides sags, a voltage swell has also been considered. In this case, the amplitude jumps from $1 \mathrm{pu}$ to 1.8 


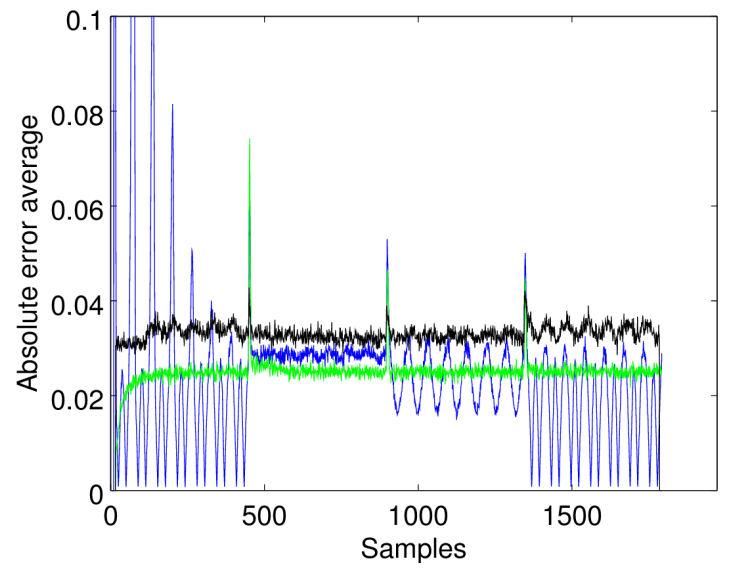

Fig. 7- Error signal for three different approaches: blockwise, for $p=15, B l=200$ and $o v l=25$, black; LMS, for $p=10, \mu=0.15$, blue; and RLS, for $p=15, \lambda=0.99$, green.

$\mathrm{pu}$, i.e. an $0.8 \mathrm{pu}$ increment in a similar fashion of the $0.8 \mathrm{pu}$ decrement for sags, although this value may not be very realistic. In general, the behaviour of the analysed method does not differ much from that shown for one step voltage sag, except for a couple of situations. First, the overdetermined block-wise approach produces a much more regular pattern. Second, some values of the parameters that control the convergence now make the algorithm diverge, when it was not the case for sags; this is due to the increment in signal magnitude, which requires lower values for step-sizes.

Regarding transients, it has to be considered that AR modelling triggers higher error at perturbation boundaries when these perturbations are a change from a sinusoidal waveform to a sinusoidal waveform, like in a sag or swell (not in a transient). Also, if the difference between perturbed and unperturbed signal is small, the algorithm will find it difficult to spot. So, for transients, the analysis of the methods indicates that:

a) The peaks are smaller than for the rest of analysed perturbations.

b) During the transient, errors are above base level.

c) Higher error does not necessary occur at transient boundaries.

\section{IEEE 1159.2 Working Group Test Waveforms}

In order to further compare the studied algorithms, test waveform 3a provided by the IEEE 1159.2 Working Group is considered (see figure 8). These test waveforms stress the influence of electronic equipment in industries, and as opposed to the synthetically generated

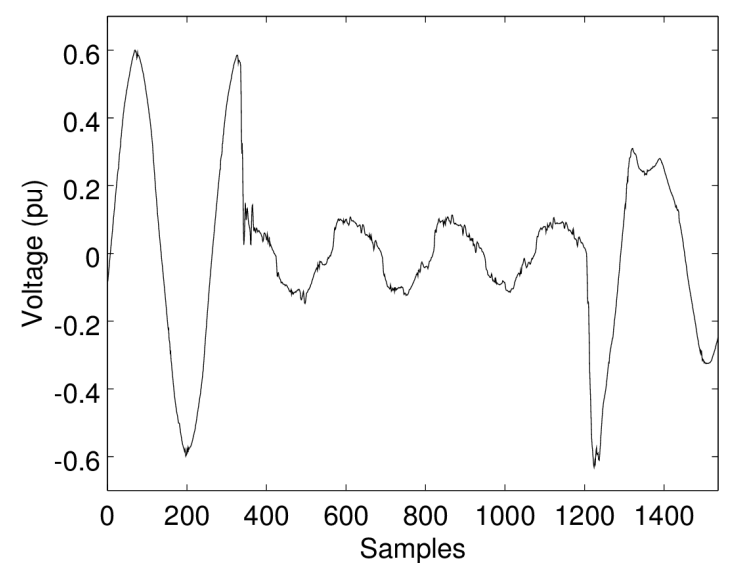

Fig. 8- Test waveform number 3 from IEEE 1159.2 working group.

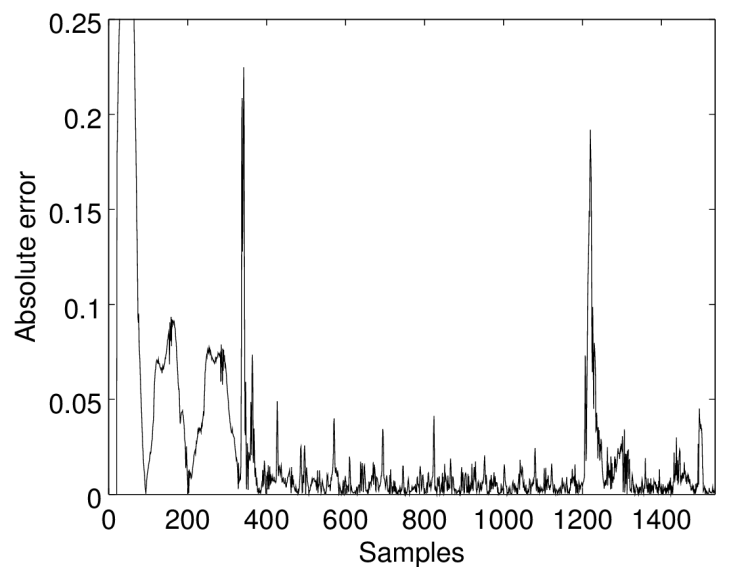

Fig. 9- Error signal of ORIV algorithm for test waveform 3a.

used previously, they show a more irregular behaviour, not only due to sags. The irregularities make the modelling process more challenging, so the modelling errors are continuously varying and peaks -indicating a sudden change in behaviour, leading to start/end of events - are more difficult to identify. This fact has even led to AR models unable to detect the power quality event if during the event itself a pattern of any kind is not followed, like it was the case for test waveforms 4 and 15.

Simulations have shown that the ORIV algorithm is the best adaptive algorithm for this particular signal (figure 9), and that in general, overdetermined implementations provide better results. Regarding the comparison against its LMS counterpart, from figure 10 it can be deduced that the latter never achieve a constant error, due to longer converging times coupled with more dynamically active voltage signal (figure 8). An overall idea of the performance of all the analysed methods can be obtained from table II. Now, from an inspection of figure 8, peaks should appear at samples 333 and 1204, where different events seem to start. 


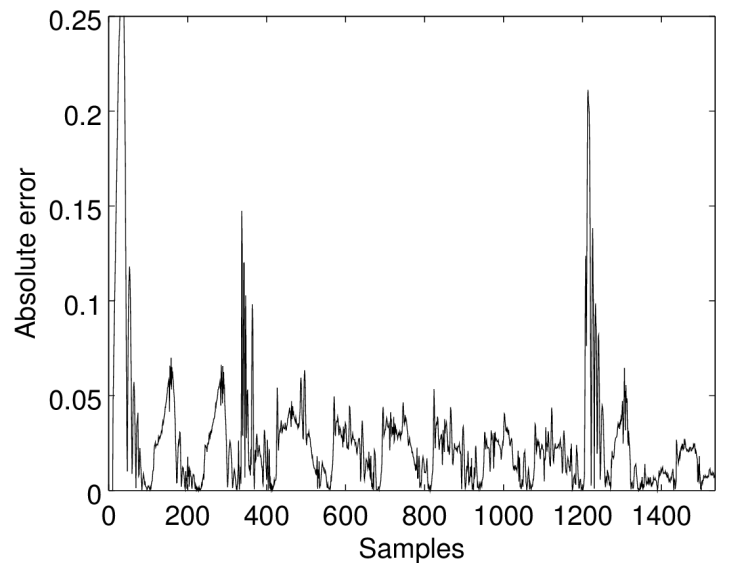

Fig. 10- Error signal of overdetermined LMS algorithm for test waveform $3 \mathrm{a}$.

Table II- Peaks location (in samples) and heights obtained during the segmentation of test waveform $3 \mathrm{a}$.

\begin{tabular}{|c|c|c|c|c|}
\hline Method & peak 1 & height 1 & peak 2 & height 2 \\
\hline Block-wise & 337 & 0.127 & 1214 & 0.072 \\
\hline $\begin{array}{c}\text { Overdetermin } \\
\text { ed block-wise }\end{array}$ & 342 & 2.26 & 1212 & 1.08 \\
\hline LMS & 342 & 0.189 & 1213 & 0.212 \\
\hline $\begin{array}{c}\text { Overdeterm } \\
\text { ined LMS }\end{array}$ & 337 & 0.147 & 1214 & 0.211 \\
\hline ORIV & 342 & 0.225 & 1220 & 0.192 \\
\hline RLS & 336 & 0.065 & 1207 & 0.033 \\
\hline
\end{tabular}

RLS algorithm provides more accurate peak locations, but the error signal it provides is far more difficult to interpret due to much lower peaks.

\section{E. Analysis}

The perturbations should be classified according to the gap happening at the boundaries of the synthetic signal used. In this work, when the jump at the boundary point is $0.68 \mathrm{pu}$, the jump is considered of sufficient entity to be easily resolved by all the studied methods. For this case, the AOGLMS algorithm provides best results, although overdetermined block-wise shows a singular behaviour. On the other hand, if the gap at the perturbation boundary is negligible, only certain methods succeed - block-wise, LMS and RLS - being the RLS the best performer. It should be noticed that the overdetermined variants do not work, maybe because they are very able to track such a small signal change, so the error is not affected. Regarding application of these methods to test waveforms obtained in industrial scenarios under power electronics equipments, analysis changes quite radically, since during the perturbation the voltage waveform shows a very irregular behaviour and hence noticeable error values can occur, concealing the peak linked to the sample point of segmentation start/end. Error signal when modelling perturbed voltage/current during a transient should be interpreted differently, since single isolated peaks are no longer present, but rather, a whole interval of high values of the error signal.

\section{Conclusions}

Adaptive algorithms, despite being lighter computationally, are able to correctly segment the perturbed signal, according to simulations. Simulations also show that the parameters that govern the algorithms must be chosen carefully to assure convergence. Besides, overdetermined approaches seem to behave more irregularly, which translates into more sharp responses to the start of the perturbed segment. So, adaptive approaches are an excellent choice for real-time segmentation, provided the signal of interest can be AR modelled except during the perturbation start/end.

\section{References}

[1] M. H. J. Bollen and I. Y.-H. Gu, Signal Processing of power quality disturbances, ser. IEEE Press Series on Power Engineering, M. E. El-Hawary, Ed. WileyInterscience, 2006.

[2] P. F. Ribeiro, C. A. Duque, P. M. Ribeiro, and A. S. Cerqueira, Power Systems Signal Processing for Smart Grids. Wiley, 2013.

[3] S. K. Singh, A. K. Goswami, and N. Sinha, "Harmonic parameter estimation of a power signal using FT-RLS algorithm," in 2014 16th International Conference on Harmonics and Quality of Power (ICHQP), May 2014, pp. 157-161.

[4] S. K. Singh, A. Nath, R. Chakraborty, J. Kalita, N. Sinha, and A. K. Goswami, "Fast transverse-RLS algorithm based power system harmonic estimation," in Information Communication and Embedded Systems (ICICES), 2014 International Conference on, Feb 2014, pp. 1-5.

[5] E. Alameda-Hernandez, F. Aznar, and G. Botella, "Comparative analysis of segmentation methods, including adaptive and hos based algorithms," in 2016 17 th International Conference on Harmonics and Quality of Power (ICHQP), Oct 2016, pp. 338-343.

[6] E. Alameda-Hernández, F. G. Montoya, M. J. MercadoVargas, G. Botella, and F. Manzano-Agugliaro, "Higher-order statistics for power systems: Effects of the sampling frequency on ergodicity," Applied Mathematical Modelling, vol. 40, no. 15-16, pp. 6924-6933, 2016. [Online]. Available: http://www.sciencedirect. com/science/article/pii/S0307904X16300683

[7] S. S. Haykin, Adaptive filter theory, 5th ed. Pearson Education, 2013.

[8] E. Alameda-Hernandez, D. Blanco, D. Ruiz, and M. Carrion, "The averaged, overdetermined, and generalized LMS algorithm," Signal Processing, IEEE Transactions on, vol. 55, no. 12, pp. $5593-5603$, dec. 2007. 